\title{
Correction to: the Video Spatial Error Concealment Algorithm Using Separately-Directional Interpolation Technique
}

\author{
Yu-Hsuan Lee ${ }^{1} \cdot$ Cheng-Hung Lin ${ }^{1} \cdot$ Chao-Chyun Chen ${ }^{1} \cdot$ Shu-Yen Lin ${ }^{1} \cdot$ Bo-Siang Huang ${ }^{1}$
}

Published online: 15 February 2020

(C) Springer Science+Business Media, LLC, part of Springer Nature 2020

\section{Correction to: J Sign Process Syst (2017) 88:13-27.} https://doi.org/10.1007/s11265-016-1112-y

I am the author of this paper published in Journal of Signal processing Systems:

Yu-Hsuan Lee, Cheng-Hung Lin, Chao-Chyun Chen, ShuYen Lin, and Bo-Siang Huang, "The Video Spatial Error Concealment Algorithm Using Separately-Directional Interpolation Technique," Journal of Signal Processing Systems, vol. 78, no. 1, pp. 13-27, Jul. 2017. (SCI, Full paper).

As a Taiwan citizen, I need to update the affiliation of the above paper, where "People's Republic of China" should be replaced by "Republic of China".

Correct affiliation should read:

Department of Electrical Engineering and Innovation Center for Big Data and Digital Convergence, Yuan-Ze University, 135 Yuan-Tung Road, Chung-Li, 32,003, Taoyuan, Taiwan, Republic of China.

The online version of the original article can be found at https://doi.org/ 10.1007/s11265-016-1112-y

Yu-Hsuan Lee

yhlee@saturn.yzu.edu.tw

Cheng-Hung Lin

chlin@saturn.yzu.edu.tw

Chao-Chyun Chen

cc.chen@saturn.yzu.edu.tw

Shu-Yen Lin

sylin@saturn.yzu.edu.tw

Bo-Siang Huang

s1004645@mail.yzu.edu.tw

1 Department of Electrical Engineering and Innovation Center for Big Data and Digital Convergence, Yuan-Ze University, 135 Yuan-Tung Road, Chung-Li, 32003 Taoyuan, Taiwan, Republic of China 